\title{
Is There a Role for Intravitreal Triamcinolone Acetonide Injection in the Treatment of Purtscher-Like Retinopathy? Report of Two Cases
}

\author{
Hasan Can Doruk, Ali Osman Saatci, Aylin Yaman \\ Department of Ophthalmology, Dokuz Eylul University, Izmir, Turkey \\ Email: hcdoruk@gmail.com
}

Received 27 September 2014; revised 27 October 2014; accepted 22 November 2014

Copyright (C) 2014 by authors and Scientific Research Publishing Inc.

This work is licensed under the Creative Commons Attribution International License (CC BY). http://creativecommons.org/licenses/by/4.0/

\section{(c) (i) Open Access}

\begin{abstract}
We hereby described two cases with unilateral Purtscher-like retinopathy. The first case occurred following an uneventful spontaneous vaginal delivery. The second case had no discernable cause despite having meticulous systemic work-up. Both eyes had low presenting visual acuity of hand movements and 20/200 consecutively. We elected to inject $1 \mathrm{mg}$ triamcinolone acetolamide intravitreally for the treatment. Though, there was some subjective visual and clinical improvement within time in both patients, we were not convinced that intravitreal injection was superior to no treatment approach.
\end{abstract}

\section{Keywords}

Purtscher's Retinopathy, Purtscher-Like Retinopathy, Triamcinolone

\section{Introduction}

Purtscher's retinopathy is an occlusive microvasculopathy associated with trauma and was first described in 1910 by Otmar Purtscher in a man who fell off a tree and suffered a head trauma [1]. The most common retinal findings are cotton-wool spots, retinal hemorrhages and Purtscher's flecken, which are considered to be pathognomonic but occur about only in $50 \%$ of patients [2]. The term of Purtscher-like retinopathy is coined for the cases with similar fundus features unassociated to any trauma.

Acute pancreatitis, chronic renal failure, hemolytic uremic syndrome, childbirth, connective tissue disorders, systemic lupus erythematosus, cryoglobulinemia, weight-lifting, battered baby syndrome and orbital injection 
are previously described causes for the Purtscher-like retinopathy [3].

At present no well-proven treatment algorithm exists for either Purtscher's or Purtscher-like retinopathy. However, several treatment modalities were employed in a small number of patients with some success. In this report, we present two patients with unilateral Purtscher-like retinopathy treated with a single $1 \mathrm{mg}$ intravitreal triamcinolone acetonide injection and discuss their clinical outcome.

\section{Case Report}

\subsection{Case 1}

A 33-year-old, gravida 2, para 2, healthy woman, who had spontaneous vaginal delivery 21 days ago was referred to us for the visual decline in her right eye. On our examination, visual acuity was 20/200 in OD and 20/20 in OS. Slit-lamp examination and intraocular pressure were normal OU. Fundoscopic examination revealed cotton-wool patches and retinal hemorrhages scattered throughout the posterior pole and peripapillary area in OD (Figure 1(a)) and the left fundus was normal. Fluorescein angiogram displayed disc staining, hypofluorescent areas corresponding to cotton wool patches and some vascular leakage in the affected eye (Figure 1(b)). OCT exhibited submacular fluid and hyperreflectivity at the ganglion cell layer due to ischemia in OD (Figure 1(c)).

Systemic examination and a meticulous laboratory evaluation including detailed hematologic tests were negative for any sistemic disease. Our diagnosis was unilateral Purtscher-like retinopathy related to the recent childbirth. Treatment options and the visual outcome were discussed with the patient and we injected $1 \mathrm{mg}$ triamcinolone acetonide intravitreally without any complication. Though the patient noted subjective visual increase, the visual acuity was still 20/200 one month after with marked improvement of fundus appearance (Figure 2(a) and Figure 2(b)). Six months later fundus and optical coherence tomography (OCT) examination looked almost normal while visual acuity was still 20/200 in OD. No injection related complication occurred during the followup.

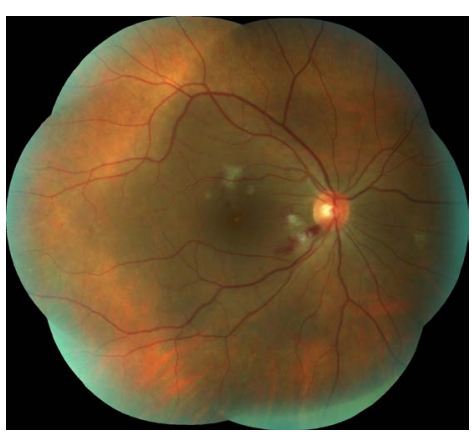

(a)

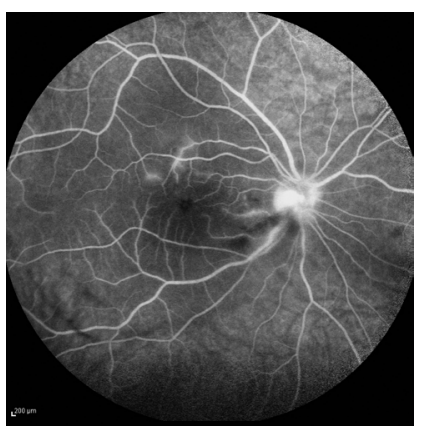

(b)

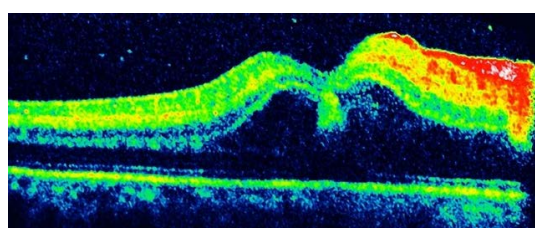

(c)

Figure 1. Case 1: right eye. (a) Color fundus; (b) Venous phase of fluorescein angiogram; (c) OCT image at the initial presentation.

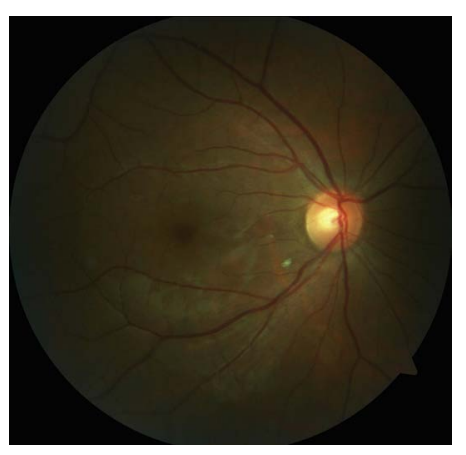

(a)

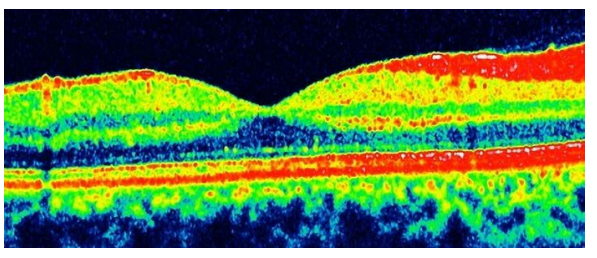

(b)

Figure 2. Case 1: right eye. (a) Color fundus picture; (b) OCT image at the first postinjection month. 


\subsection{Case 2}

A 60-year-old woman was referred to us with a recent visual decline in her left eye. Her visual acuity vas 20/60 OD and HM OS. Slit-lamp examination and intraocular pressure measurement were normal. While fundoscopic examination showed signs of chronic central retinal vein occlusion in her right eye, cotton-wool exudates and some peripapillary intraretinal hemorrhages were seen in the left fundus (Figure 3(a) and Figure 3(b)). OCT demonstrated cystic macular edema in OD and some hyperreflectivity in the ganglion cell layer at the left eye (Figure 3(c) and Figure 3(d)).

Fluorescein angiogram showed cystoid macular edema in OD and some peripapillary hypofluorescent areas corresponding to cotton-wool spots (Figure 3(e) and Figure 3(f)). She reported that she had several bevacizumab injections for her right eye elsewhere almost three years ago without any significant visual gain. Our diagnosis was right old CRVO and left unilateral Purtscher-like retinopathy. Full systemic and hematologic work-up were performed without any positive finding. After being advised of the risks and benefits, the patient elected to undergo intravitreal injection of $1 \mathrm{mg}$ triamcinolone acetonide OS. Though the patient was satisfied with the visual outcome her visual acuity was counting fingers at 2 meters in OS one week after the injection with some improvement in fundus appearance (Figure 4). Eight months after the uneventful injection, her visual acuity was still 20/200 in OS with slight left disc pallor (Figure 5).

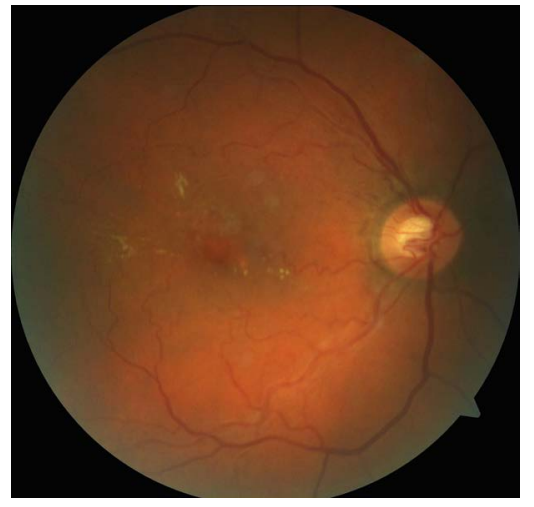

(a)

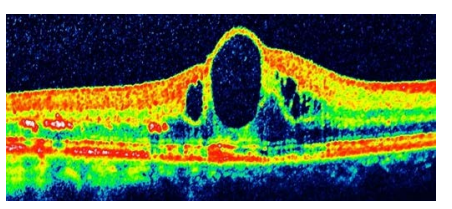

(c)

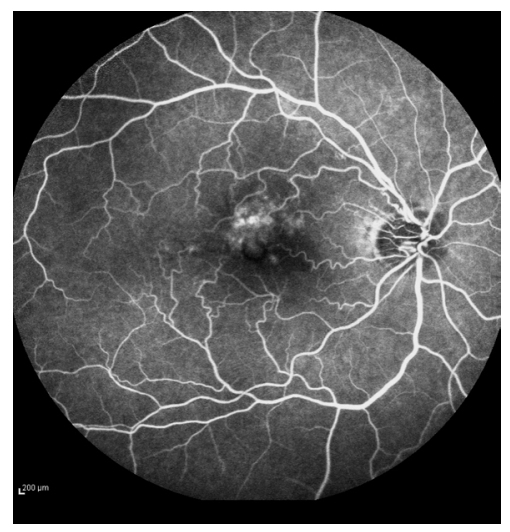

(e)

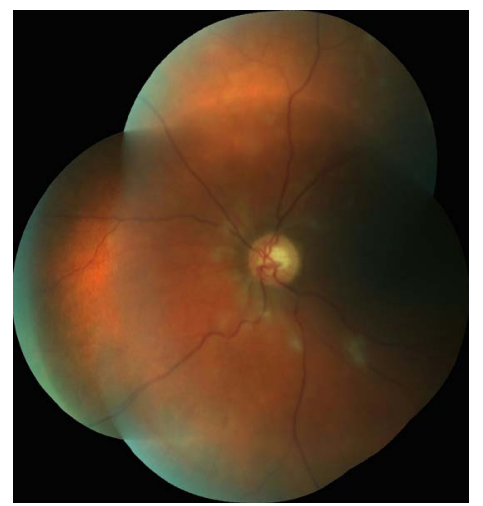

(b)

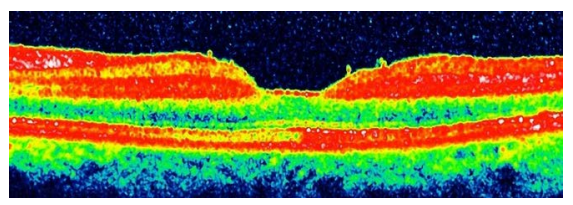

(d)

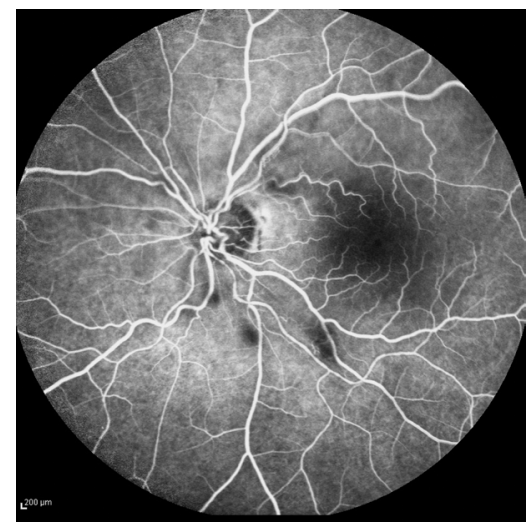

(f)

Figure 3. Case 2. (a) Right eye, color fundus picture; (b) Left eye, color fundus picture; (c) Right eye, OCT image; (d) Left eye, OCT image; (e) Right eye, venous phase of fluorescein angiogram at the initial presentation; (f) Left eye, venous phase of fluorescein angiogram at the initial presentation. 


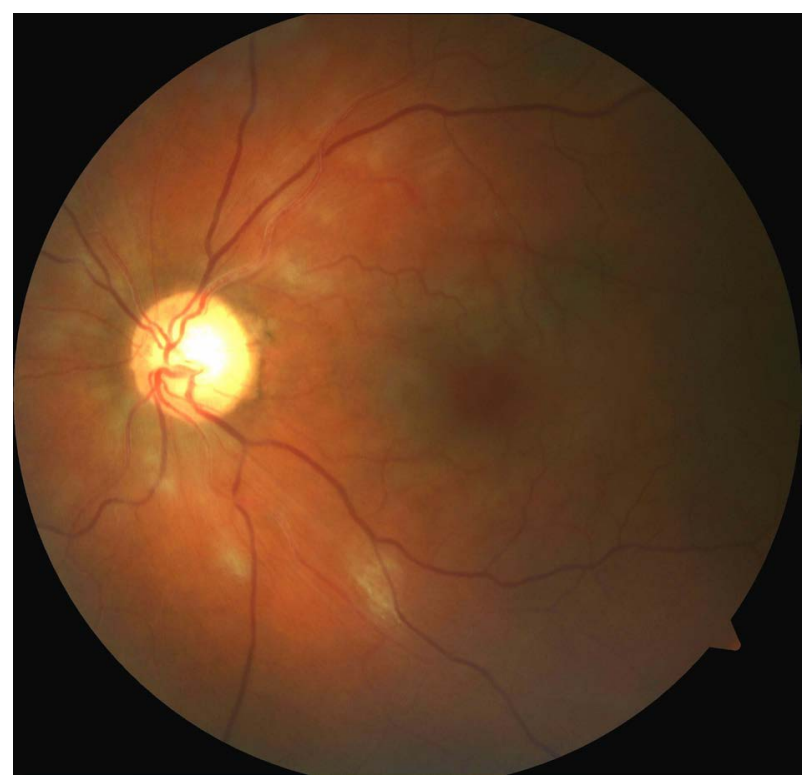

Figure 4. Case 2, left eye, color fundus picture at the first post injection week.

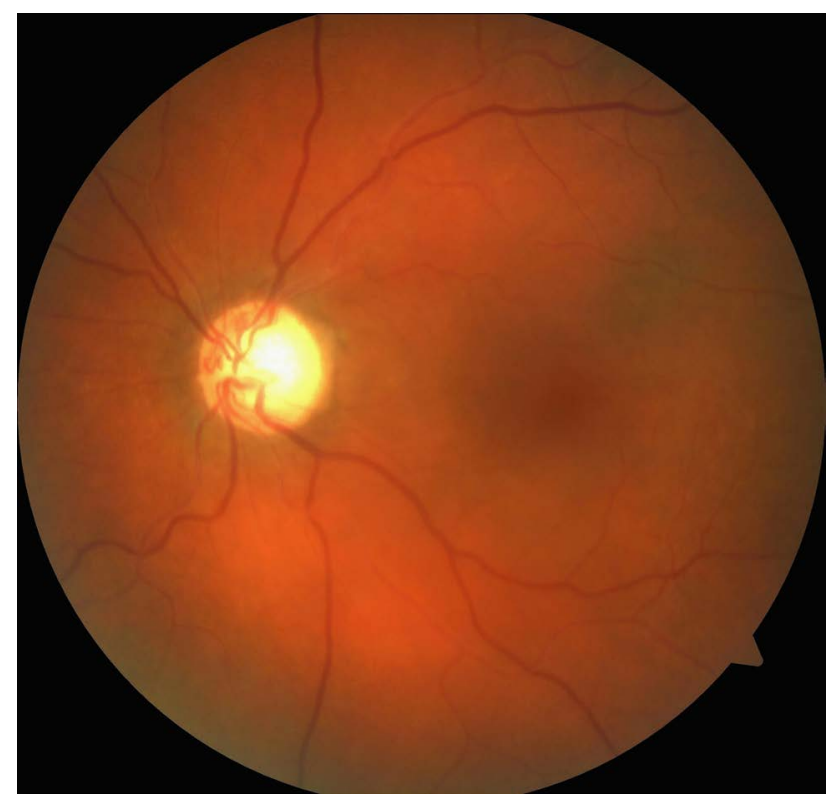

Figure 5. Case 2, left eye, color fundus picture at the eighth post injection month.

\section{Discussion}

The diagnosis of Purtscher's and Purtscher-like retinopathies is mostly based on the clinical findings and past medical history. A histopathologic study showed edema of inner retinal layers, partial loss of the nerve fiber, ganglion cell and inner plexiform layers and abrupt transition to normal retina from the affected area [4]. However, the pathophysiology of the injury is still under debate.

Several treatment modalities were contemplated in cases with Purtscher-like retinopathy. While some physcians tend to observe expectantly, others primarily administer mostly systemic corticosteroids [2]. On the other hand, most cases resolve spontaneously one to three months after the resolution of underlying systemic problem. It is important to know that several factors were deemed to have prognostic value for the poor visual outcome 
such as optic disc swelling or disc leakage seen on fluorescein angiography, choroidal hypoperfusion and involvement of the outer retina, retinal capillary nonperfusion and a prior episode of Purtscher's retinopathy [3].

The most preferred treatment option is systemic megadose steroid administration. The plausible rationale for this regimen is to block complement-mediated leucocyte aggregation and inhibit oxygen-free radicals' formation [5] [6]. While some authors reported good visual results with pulse steroid treatment others presented cases without any satisfactory visual improvement, following the treatment. Wang et al. [7] reported that a patient with unilateral blurred vision in the right eye following a traffic accident occurred two days prior to visual deterioration. With the classical fundus appearance, the diagnosis of unilateral Purtscher's retinopathy was established and intravenous megadose steroid therapy comprised of methylprednisolone $250 \mathrm{mg}$ every six hours for 3 days was employed. The visual acuity improved to 6/12 from CF in the right eye with the resolution of fundus appearance at the first month. Atabay et al. [8] described a case with late visual recovery managed with systemic steroids who diagnosed with Purtscher's retinopathy. Initially patient was treated with $60 \mathrm{mg}$ oral prednisolone and 3 weeks later visual acuity was still 20/800. Then they switched to intravenous high-dose steroids and surprisingly vision improved to 20/70 just after 3 days. They attributed this visual gain to the ability of high-dose steroids to stabilize the damaged neuronal membrane and microvascular channels, enabling partial recovery of nerve-fibers. Zwolinska et al. [9] described a 4-year-old male child with nephrotic syndrome and bilateral Purtscher-like retinopathy. He was treated with intravenous methylprednisolone, $20 \%$ albumin infusion and low molecular weight heparin. His visual acuity improved from CF at $2.5 \mathrm{~m}$ to 9/10 at the third day and ischemic retinal blanching and associated hemorrhages showed resolution mostly within two weeks. Cumurcu et al. [10] described a unilateral Purtscher retinopathy complicating mandibular fracture in an 11-year-old child one day after the initial trauma. Despite pulse steroid therapy for three days and consecutive oral steroid administration no visual improvement was noted. In another study, Yan Yan and Xi Shen [11] reported a case of bilateral Purtscher-like retinopathy in an 18-year-old female patient with dermatomyositis. Despite the initial treatment of intravenous methylprednisolone, vision loss progressed over the next three months. Bilateral optic disc pallor attenuated retinal arteries and mottled RPE appearance ensued during the course.

Systemic steroids are fraught with their well-known several serious systemic complications. In the group of patients with Purtscher-like retinopathy already having coexisting systemic problems, systemic steroid administration may aggravate these steroid-induced complications. Thereby, local steroid administrations may be helpful to avoid the systemic steroid side effects. Olson et al. [12] performed subtenon injection of $20 \mathrm{mg}$ triamcinolone in the right eye of a patient with bilateral Purtscher-like retinopathy observed ten days after the childbirth. Five weeks following the injection, the visual acuity improved from 20/200 to 20/60. OCT examination showed $30 \%$ reduction in the central macular thickness. However, better seeing uninjected eye exhibited only $14 \%$ reduction in central macular thickness.

To our knowledge, no report exists about the place of intravitreal triamcinolone injection in Purtscher-like retinopathy. We decided to treat the study patients as both had poor initial visual acuity and unilateral involvement. However, in order to avoid systemic steroid side-effects we preferred to administer intravitreal triamcinolone acetonide. Instead of $4 \mathrm{mg}$ dose, we chose $1 \mathrm{mg}$ dose as the volume of injection was smaller and the possible intraocular pressure hike sometimes seen after any injection was less likely. Though both patients were satisfied with the visual outcome, we were not convinced enough that there was any treatment benefit over the natural disease course. Still intravitreal triamcinolone acetonide or even dexamethasone implant administration may find a niche in the treatment armamentarium of some cases with Purtscher-like retinopathy.

\section{References}

[1] Purtscher, O. (1910) Noch Unbekannte Befunde Nach Schadeltrauma. Bericht Uber Die Zusammenkunft Der Deutschen Ophthalmologischen Gesellschaft, 36, 294-301.

[2] Miguel, A.I.M., Henriques, F., Azevedo, L.F.R., Lourerio, A.J. and Maberley, D.A.L. (2013) Systematic Review of Purtscher's and Purtscher-Like Retinopathies. Eye, 27, 1-13. http://dx.doi.org/10.1038/eye.2012.222

[3] Agrawal, A. and McKibbin, M.A. (2006) Purtscher's and Purtscher-Like Retinopathies: A Review. Survey of Ophthalmology, 51, 129-136. http://dx.doi.org/10.1016/j.survophthal.2005.12.003

[4] Kincaid, M.C., Green, W.R., Knox, D.L. and Mohler, C. (1982) A Clinicopathological Case Report of Retinopathy of Pancreatitis. British Journal of Ophthalmology, 66, 219-226. http://dx.doi.org/10.1136/bjo.66.4.219

[5] Hammerschmidt, D.E., White, J.G., Craddock, P.R. and Jacob, H.S. (1979) Corticosteroids Inhibit Complement-Induced Granulocyte Aggregation. A Possible Mechanism for Their Efficacy in Shock States. Journal of Clinical In- 
vestigation, 63, 798-803. http://dx.doi.org/10.1172/JCI109365

[6] Goldstein, I.M., Roos, D., Weisman, G. and Kaplan, H.B. (1976) Influence of Corticosteroid on Human Polymorphonuclear Leukocyte Function in Vitro: Reaction of Lysosomal Enzyme Release and Superoxide Production. Inflammation, 1, 305-315. http://dx.doi.org/10.1007/BF00917870

[7] Wang, A.G., Yen, M.Y. and Liu, J.H. (1998) Pathogenesis and Neuroprotective Treatment in Purtscher's Retinopathy. Japanese Journal of Ophthalmology, 42, 318-322. http://dx.doi.org/10.1016/S0021-5155(98)00015-X

[8] Atabay, C., Kansu, T. and Nurlu, G. (1993) Late Visual Recovery after Intravenous Methylprednisolone Treatment of Purtscher's Retinopathy. Annals of Ophthalmology, 25, 330-333.

[9] Zwolińska, D., Medyńska, A., Galar, A. and Turno, A. (2000) Purtscher-Like Retinopathy in Nephrotic Syndrome Associated with Mild Chronic Renal Failure. Pediatric Nephrology, 15, 82-84.

[10] Cumurcu, T., Yardım, H., Güven, M., Kılıç, R. and Ozkan, N. (2008) Purtscher’s Retinopathy: An Unusual Association with a Complicated Mandibular Fracture. Turkish Journal of Medical Sciences, 38, 373-376.

[11] Yan, Y. and Shen, X. (2013) Purtscher-Like Retinopathy Associated with Dermatomyositis. BMC Ophthalmology, 13, 36. http://dx.doi.org/10.1186/1471-2415-13-36

[12] Olson, J., Rouhani, B. and Mandava, N. (2008) Sub-Tenon’s Triamcinolone for Post-Partum Purtscher’s-Like Retinopathy. Clinical Ophthalmology, 2, 195-198. http://dx.doi.org/10.2147/OPTH.S2080 
Scientific Research Publishing (SCIRP) is one of the largest Open Access journal publishers. It is currently publishing more than 200 open access, online, peer-reviewed journals covering a wide range of academic disciplines. SCIRP serves the worldwide academic communities and contributes to the progress and application of science with its publication.

Other selected journals from SCIRP are listed as below. Submit your manuscript to us via either submit@scirp.org or Online Submission Portal.
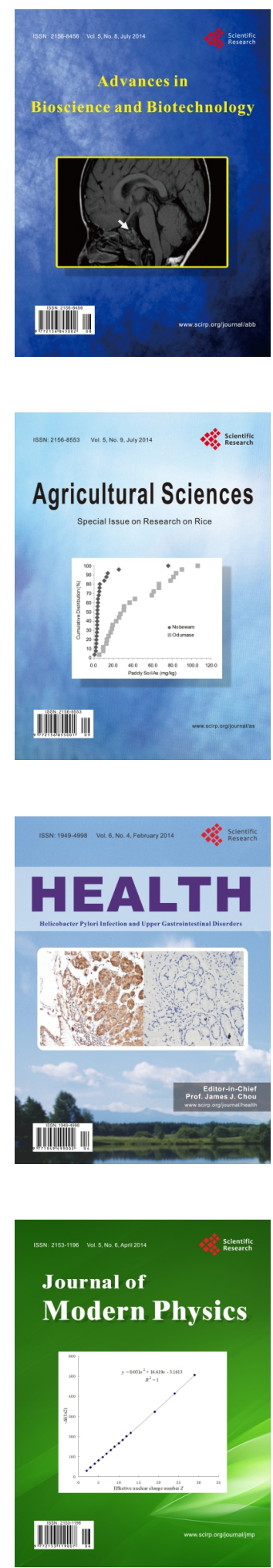
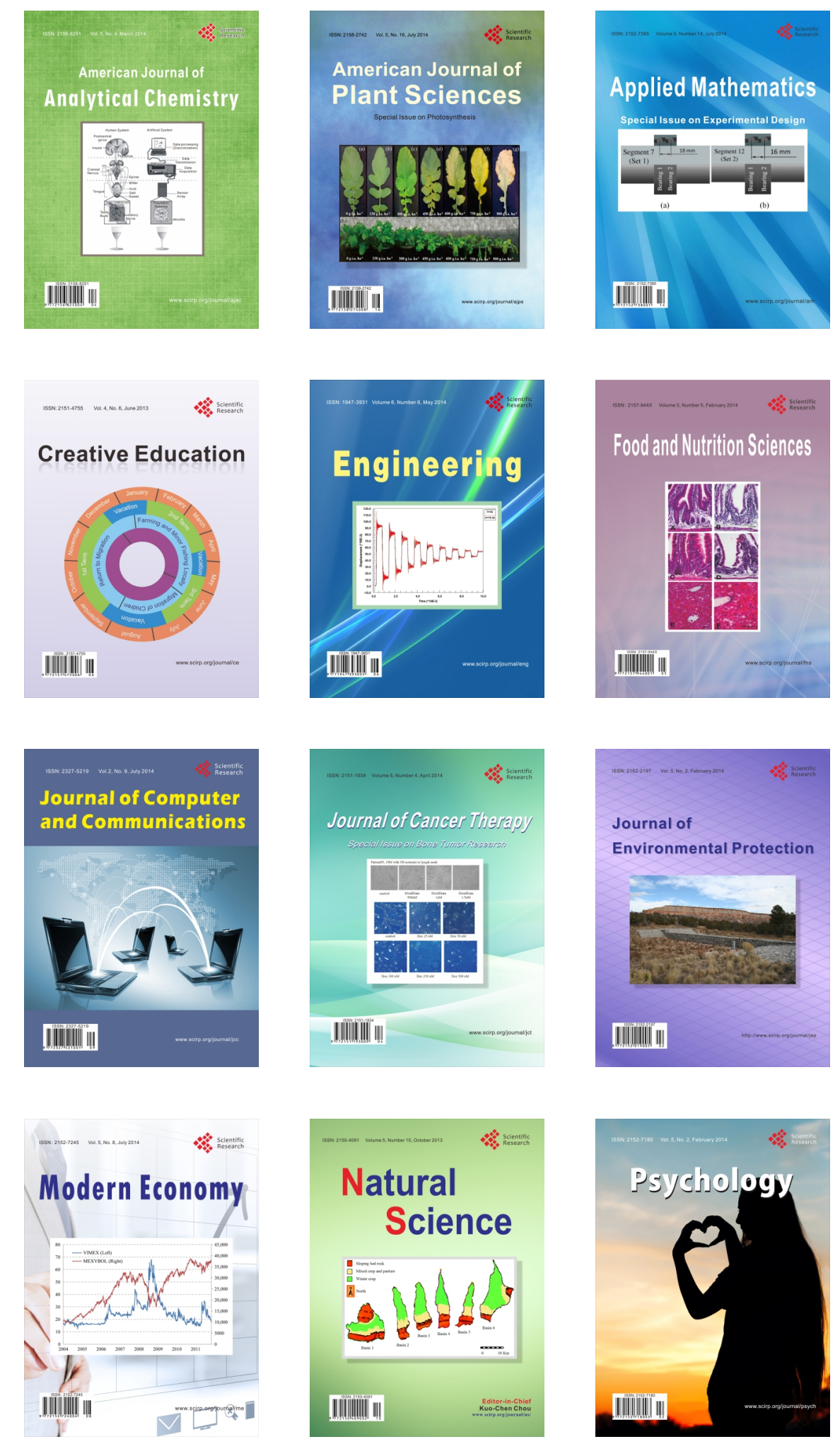